\title{
A FULLY EMPIRICAL APPROACH TO GALAXY EVOLUTION
}

\author{
Alvio Renzini \\ INAF - Osservatorio Astronomico di Padova, Vicolo dell'Osservatorio 5, I-35122 Padova, Italy \\ E-mail: alvio.renzini@oapd.inaf.it \\ (Received July 1, 2010; Accepted August 19, 2010)
}

\begin{abstract}
Observations of large samples of galaxies from low to high redshifts are composing a picture of remarkable simplicity: (1) The star formation rate (SFR) of starforming galaxies scales almost linearly with mass, strongly decline with cosmic time, and exhibits very small scatter around the average relation. (2) Due to the high observed SFRs the mass of galaxies at high redshifts must increase very rapidly, and yet the mass function of star forming galaxies evolves only very slightly with redshift. (3) At all redshifts the fraction of quenched (passively evolving) galaxies increases with galactic stellar mass and with local overdensity, with the remarkable property that the relative efficiency of "mass quenching" is independent of environment, and that of "environment quenching" is independent of mass. In a recent paper by the zCOSMOS collaboration, Peng et al. (2010) demonstrate that these three empirical facts suffice to account for the observed evolution of the galaxy mass function and naturally generate the "double-Schechter" mass function for quenched galaxies.
\end{abstract}

key words: galaxies: formation; galaxies: evolution; stars: formation

\section{INTRODUCTION}

In the most common approach to galaxy formation and evolution the results of semi-analytic models or hydrodynamical simulations are compared to observations, checking to which extent the models fit or do not fit the data. Usually they don't fit so well, and therefore parameters in the theoretical models are adjusted in the attempt to improve the fit, thus venturing into the next iteration.

Multiwavelength surveys of galaxies are now delivering an unprecedented wealth of data, with fairly representative slices of the Universe at various redshifts. Thus, another, fully empirical, phenomenological approach becomes possible for the first time: one in which evolutionary links and trends are derived directly from the data, without dealing at all with the complexities of the numerous physical processes that indeed must govern galaxy evolution. This is quite a different point of view over galaxy evolution, compared to that which has been customary in all recent years. To be fully ap- preciated it requires, so for saying, a mental change of coordinates with respect to our previous attitude. For example, restraining temporarily from searching for the underlying physics, and just explore what data themselves demand.

It was a pleasure for me to report some exciting results of such phenomenological approach to the Galaxy Formation Forum celebrating the $125^{\text {th }}$ birthday of the Yonsei University. Such results have been achieved in the frame of the zCOSMOS collaboration (Lilly et al., 2009) and are fully reported in Peng et al. (2010).

\section{LATEST RESULTS}

At first sight the population of galaxies appears to host an infinite variety of types and subtypes, of peculiar objects making a taxonomist's nightmare. However, when large samples of galaxies are culled and studied, it appears that the vast majority of galaxies, at all redshifts, follow simple scaling relations, while outliers represent 


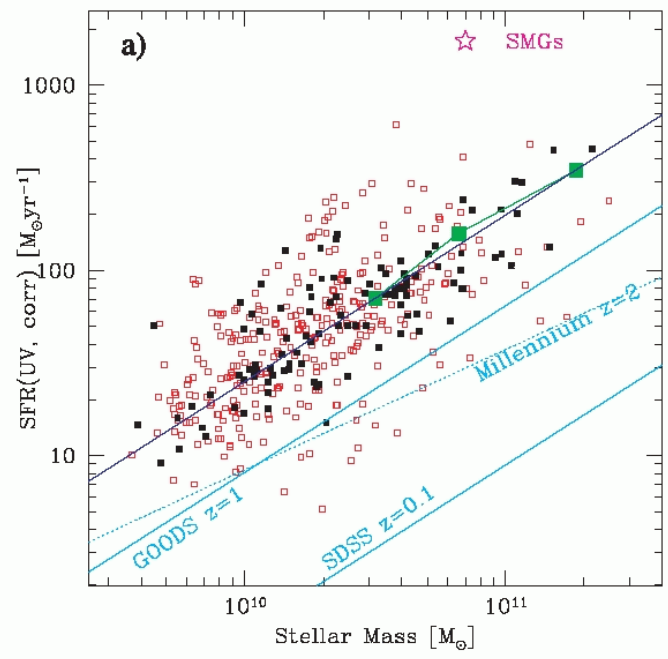

Fig 1. - The star formation rate vs. stellar mass (respectively in $\mathrm{M}_{\odot} \mathrm{yr}^{-1}$ and $\mathrm{M}_{\odot}$ ) for star-forming galaxies at $1.4<z<2.5$ in the GOODS-South field (adopted from Daddi et al., 2007). The SFR was derived from the UV flux plus extinction correction. The mean SFR-mass relation at $z=1$ and $z=0.1$ are also shown. The green square show the SFRmass relation obtained by using the radio luminosity as a SFR indicator.

a tiny minority.

Thus, the Peng et al. phenomenological model is based on three remarkable simplicities, namely:

- The tight relation between the star formation rate (SFR) of star-forming galaxies, their mass, and the cosmic epoch:

$$
S F R \simeq 250 \frac{M_{\star}}{10^{11} \mathrm{M}_{\odot}}\left(\frac{t}{3.5 \mathrm{Gyr}}\right)^{-2.2}\left(\mathrm{M}_{\odot} / \mathrm{yr}\right)
$$

where $M_{\star}$ is the galaxy stellar mass and $t$ the cosmic time (Daddi et al., 2007; Elbaz et al., 2007; Noeske et al., 2007; Brinchmann et al., 2004; Pannella et al., 2009) which holds up to $z \sim 2-2.5$. Beyond this redshift, the specific SFR ( $\equiv \mathrm{SFR} / M_{\star}$ ) appears to flatten, and remains nearly constant all the way to very high redshifts (Gonzalez et al., 2010).

- The $M^{*}$ and $\alpha^{*}$ in the Schechter mass function of star-forming galaxies are remarkably constant, while the normalization $\phi^{*}$ increases slightly with time (Pozzetti et al., 2009; Ilbert et al., 2010).

- The fraction of passively evolving (red) galaxies is a strong function of both stellar mass and environment (local overdensity), but the differential effect of mass and environment on the quenching of star formation in galaxies are fully separable, i.e., the relative effect of mass in the quenching of SF is independent of environment, and the relative effect of environment is independent of mass (Peng et al., 2010). This demonstrates that two distinct processes govern the quenching of SF in galaxies.

A few figures illustrate the three founding facts of this empirical approach. Figure 1 shows the SFR vs mass relation at $z \sim 2$ from Daddi et al. (2007) and Figure 2 the same relation at $z \sim 0.1$ as derived by Peng et al. from SDSS data. Figure 3 shows the (mild) evolution of the mass function of star-forming galaxies from Ilbert et al. (2010). Finally, the third remarkable simplicity, the fraction of quenched galaxies as a function of mass and environment is illustrated by Figure 4. Clearly, mass matters for quenching, as does the environment, but they do so independent of one another.

Equation (1) demands an extremely fast, quasiexponential growth of galaxy mass around $z \sim 2$, which would lead to an enormous overgrowth of galaxies, if not interrupted by SF quenching turning galaxies from being SF to passively evolving (Renzini, 2009). Indeed, the apparent contradiction of the two first points above, the high SFRs and the invariance of the mass function, demand quenching. Were we blind to passive galaxies, those two empirical facts would suffice to demonstrate to us the existence of quenched galaxies!

Equation (1) together with the separability of mass quenching and environment quenching give us the key to phenomenologically describe as a function of redshift (cosmic time) the growth of galaxies and the rate at which they are quenched. How this is implemented in Peng et al. (2010) cannot be described here in detail without virtually replicating in full the same paper. It is sufficient to mention here the main lines and results.

In practice, while the mass growth follows Equation (1), a sort of continuity equation is solved for the quenching rate of galaxies:

$$
\frac{d N_{\mathrm{q}}}{d t}=\frac{\mathrm{SFR}}{M^{*}}+f(\rho, t)+\kappa,
$$

where $N_{\mathrm{q}}$ is the volume number density of quenched galaxies, the first term represent the mass quenching probability (independent of environment), the second term the environment quenching probability (independent of mass, see Peng et al.) and the last term is 


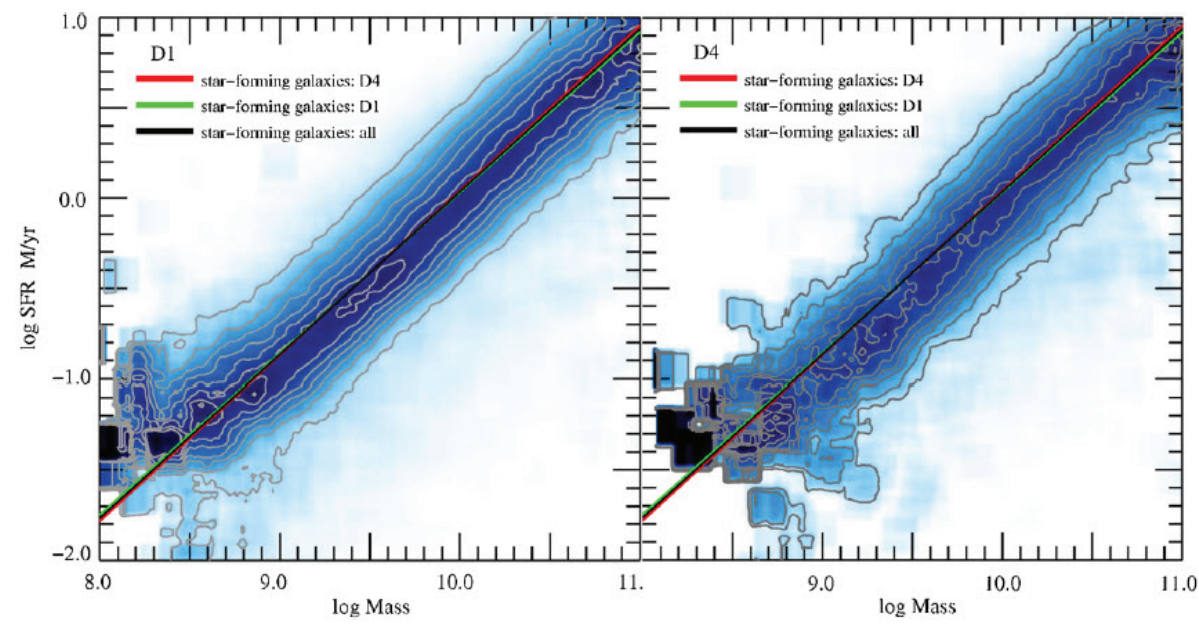

Fig 2.- The SFR-mass relation for star-forming galaxies in the local Universe from the SDSS data with the SFR derived from $\mathrm{H} \alpha$ (adopted from Peng et al., 2010). The left panel shows the relation for the lowest environmental density quartile and the right panel for the highest density quartile. Note how the SFR is the remarkably insensitive to local overdensity. The slope of the best fit SFR-mass relation is $\sim 0.9$.

meant to describe a quenching probability due to merging (assumed independent of mass). Note that the mass quenching term is here proportional to the SFR, as indeed the SFR is proportional to mass from Equation (1). The empirical evidence is that as mass grows via star formation and the SFR increases along with it, so does the probability for a galaxy to get quenched.

The result can be most effectively visualized by looking at the movie that can be downloaded from the URL:

www.exp-astro.phys.ethz.ch/zCOSMOS/MF_simulation_d1_d4.mov

which shows the evolution of the mass functions of galaxies and the quenched fraction through cosmic time, starting from a seed distribution at very high redshift, and does so separately for the low and high density regions.

Figure 5 visualizes the main results of this empirical model of galaxy evolution, which successfully reproduces several key feature of the present galaxy populations in low and high density environments, and that can be summarized as follows:

- The model establishes the Schechter function of star-forming galaxies, and the constancy of its $M^{*}$ from at least $z \sim 2$ to the present.

- It also establishes the double Schechter function which is characteristic of passive (quenched) galaxies, which results from having two indepen- dent quenching processes, one controlled only by mass and one controlled only by environment.

- It produces precisely the local set of values of the Schechter parameters $\left(M^{*}, \phi^{*}, \alpha^{*}\right)$ for the mass functions of star-forming as well as quenched galaxies.

- It reproduces the fraction of quenched galaxies in different environments.

- It qualitatively explains several other trends with mass of the stellar populations in quenched galaxies, such as average age, $\alpha$-element enhancement, and others.

\section{CONCLUSION}

I believe that with Peng et al. a major step forward has been made towards a systematic understanding of galaxy evolution, yet this still is a purely phenomenological model. There is no physics. At the onset of the era of precision (concordance) cosmology somebody said Now that the proper stage has been set up, what we need is a good play. With Peng et al. phenomenological model we don't have the play yet, just the plot, and a fairly good one. For the full play we need to introduce actors, i.e., the real physical processes at work, and force them to strictly follow the plot. 


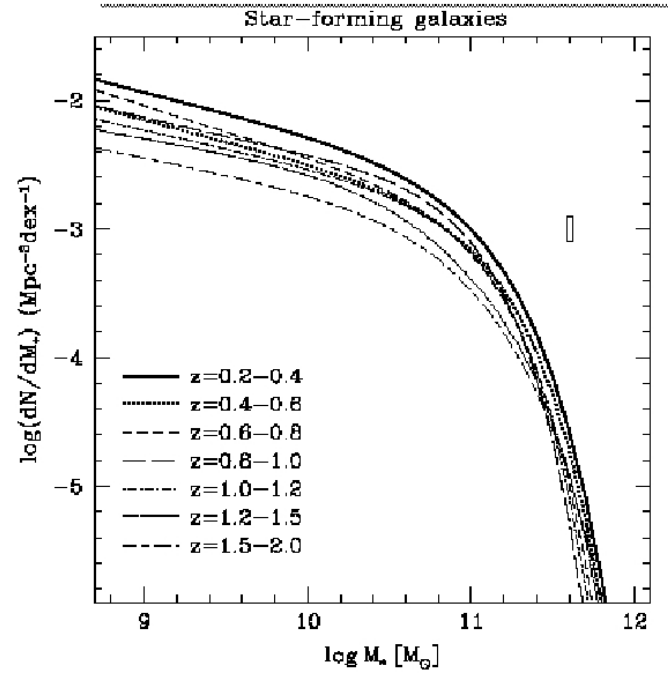

Fig 3.- The evolution up to $z \simeq 2$ of the mass function of star-forming galaxies (adopted from Ilbert et al., 2010). Note that the corresponding $M^{*}$ in the Schechter fits is remarkably constant through the whole redshift range.

The company for the play includes many actors, and several have primadonna ambitions. Among them star formation, galactic winds, cold streams, AGN feedback, the IMF, clump physics, supernova feedback, chemical evolution, black hole formation, strangulation, mergers, starbursts, disk instabilities, ram pressure, and more. Quite a turbulent assembly of actors for the theoretical impresarios to orchestrate! We can only wish good luck to them.

\section{ACKNOWLEDGEMENTS}

I wish to thank the Department of Astronomy of the Yonsei University for having offered the opportunity to attend the Galaxy Formation Forum at which this note was presented.

\section{REFERENCES}

Brinchmann, J., et al., 2004, The Physical Properties of Star-Forming Galaxies in the Low-Redshift Universe, MNRAS, 351, 1151

Daddi, E., et al., 2007, Multiwavelength Study of Massive Galaxies at $\mathrm{z} \sim 2$. I. Star Formation and Galaxy Growth, ApJ, 670, 156

Elbaz, D., et al., 2007, The Reversal of the Star Formation-Density Relation in the Distant Universe, A\&A, 468, 33

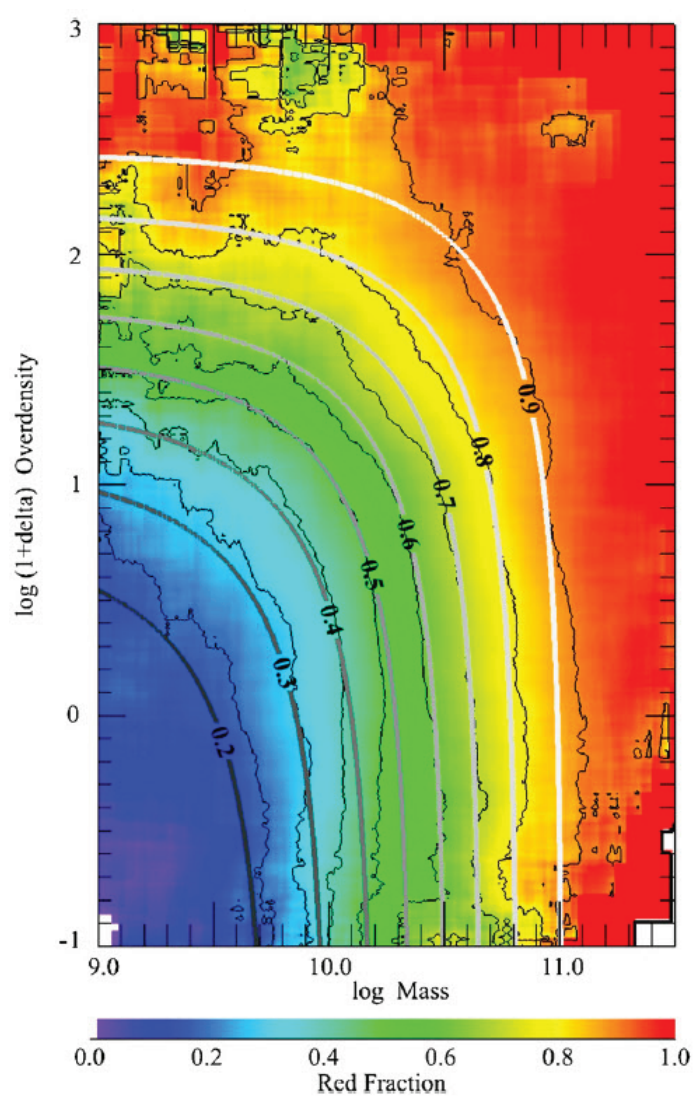

Fig 4. - The fraction of quenched (red) galaxies in SDSS as a function of stellar mass and environment (adopted from Peng et al., 2010).

Gonzalez, V., et al., 2010, The Stellar Mass Density and Specific Star Formation Rate of the Universe at $\mathrm{z} \sim 7$, ApJ, 713, 115

Ilbert, O., et al., 2010, Galaxy Stellar Mass Assembly between $0.2<z<2$ from the S-COSMOS Survey, ApJ, 709, 644

Lilly, S. J., et al., 2009, The zCOSMOS 10k-Bright Spectroscopic Sample, ApJS, 184, 218

Noeske, K. G., et al., 2007, Star Formation in AEGIS Field Galaxies Since $z=1.1$ : The Dominance of Gradually Declining Star Formation, and the Main Sequence of Star-Forming Galaxies, ApJ, 660, L43

Pannella, M., et al., 2009, Star Formation and Dust Obscuration at $\mathrm{z} \sim 2$ : Galaxies at the Dawn of Downsizing, ApJ, 698, L116

Peng, Y., et al., 2010, Mass and Environment as Drivers of Galaxy Evolution in SDSS and zCOSMOS and the Origin of the Schechter Function, ApJ, 721, 193 


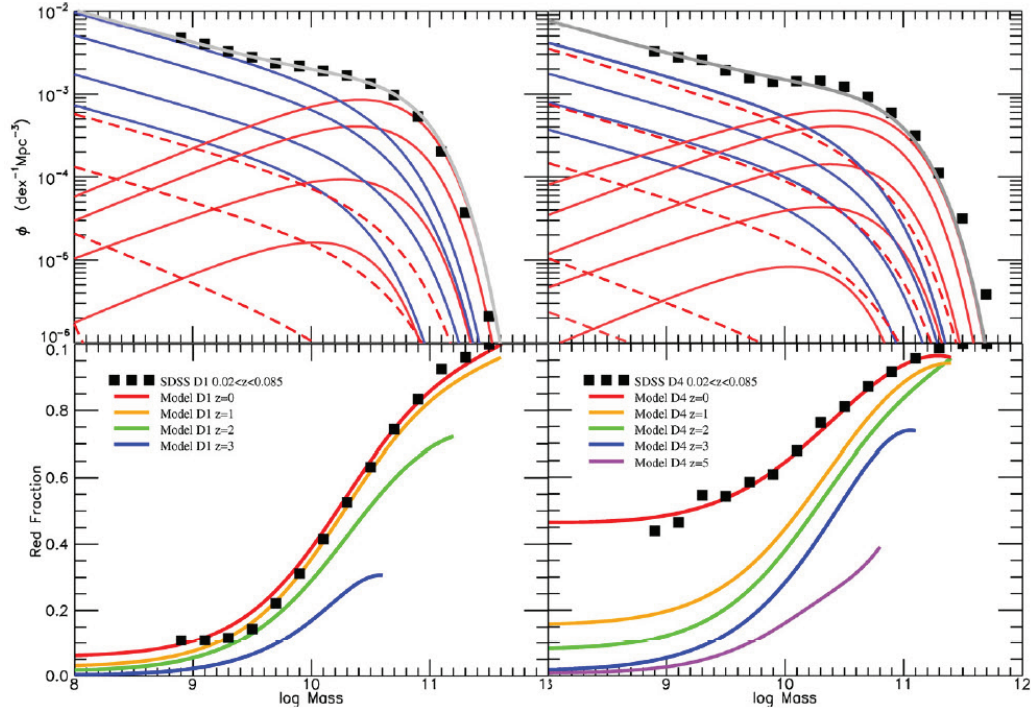

Fig 5.- The evolution of the mass functions (upper panels) and the quenched (red) fraction (lower panels), respectively in the low density quartile (left panels) and high density quartile (right panels). Red solid lines show the evolution of the mass function of quenched galaxies that are mass-quenched, while red dashed lines refer to galaxies that are environment-quenched. The blue lines show the evolution of the mass function of star-forming galaxies. Mass functions are shown bottom-up for $z=3,2,1,0$, and also for $z=5$ in the right panel. The top gray line is the final, cumulative mass function (SF + quenched galaxies) of the model, with overplotted the empirical mass function from SDSS data (black squares). Lower panels include the definition of the various lines and symbols. This figure is adopted from Peng et al. (2010).

Pozzetti, L., et al., 2009, zCOSMOS - 10k-Bright Spectroscopic Sample. The Bimodality in the Galaxy Stellar Mass Function: Exploring Its Evolution with Redshift, A\&A, submitted, arXiv:0907.5416

Renzini, A., 2009, A Different Approach to Galaxy Evolution, MNRAS, 398, L58 